Artículo Original

\title{
Eficiencia de las zanjas de infiltración tipo Niimi y normal en el tratamiento de aguas residuales domesticas en Villa los Sauces, Lima (Perú)
}

\section{EFFICIENCY OF INFILTRATION DITCHES NIIMI TYPE AND NORMAL IN THE TREATMENT OF DOMESTIC WASTEWATER IN VILLA LOS SAUCES, LIMA (PERU)}

\author{
JHOENMERT TINOCO CANTO§*, DEYSI LAURA PEZO§, MILDA CRUZ HUARANGA§, \\ ANGEL RAMIREZ GARCIA§
}

Recibido: 15 septiembre de 2018 / Aceptado: 07 noviembre de 2018

$\S E P$. Ingeniería Ambiental, Facultad de Ingeniería y Arquitectura, Universidad Peruana Unión, Perú.

\begin{abstract}
Resumen
Nuestra investigación tuvo el objetivo de determinar la eficiencia de las zanjas de infiltración tipo Niimi y Normal en el tratamiento de aguas residuales domesticas en Villa los Sauces - Lurigancho. Se construyó un tanque de abastecimiento, añadido de una cámara séptica conectada a una caja de distribución. Para ambos sistemas se insertó en la base un tubo de PVC de 3/4 de $3 \mathrm{~m}$ con orificios de $10 \mathrm{~mm}$ con separación de 250 $\mathrm{cm}$, por donde se obtuvo el efluente del agua tratada. En ambos sistemas se utilizaron una capa de arena y una capa de grava en el fondo, ambos de $10 \mathrm{~cm}$. El funcionamiento inició en la cámara séptica donde se realizó el tratamiento primario con procesos bioquímicos, luego de ingresar a las zanjas las aguas pasaron por un tratamiento secundario donde fueron filtradas por la grava y por la arena adhiriéndose a estos los coloides, generando la disminución de la turbiedad y por ende de los microorganismos. Al tener contacto con el suelo, los patógenos del agua residual murieron por los cambios de temperatura y humedad, también por falta de nutrientes, acción de los hongos y consumo por los protozoos. El agua tratada en ambas zanjas no superó los límites máximos permisibles (LMP) según el D.S. N $^{\circ}$ 003-2010-MINAM y estuvo dentro de los estándares de calidad ambiental (ECA) del D.S. N ${ }^{\circ}$ 015-2015-MINAM respecto a los valores de DBO, DQO, conductividad, $\mathrm{pH}$, Oxígeno Disuelto y Temperatura. Lo coliformes totales y termotolerantes se redujeron en un $90 \%$. La eficiencia de la zanja tipo Niimi fue del $70 \%$ y la zanja normal fue de $61 \%$. El agua tratada por ambos sistemas cumplieron con los LMPs y ECAs sin embargo la zanja tipo Niimi fue más eficiente. Palabras clave: aguas, tratamiento, nutrientes, calidad, microorganismos.
\end{abstract}

\section{Abstract}

Our research had the objective of determining the efficiency of Niimi and Normal type infiltration ditches in domestic wastewater treatment in Villa los Sauces - Lurigancho. A supply tank was built, adding a septic chamber connected to a distribution box. For both systems, a $3 \mathrm{~m}$ tubo PVC tube with $10 \mathrm{~mm}$ holes with $250 \mathrm{~cm}$ separation was inserted into the base, where the treated water effluent was obtained. In both systems a layer of sand and a layer of gravel were used in the bottom, both of $10 \mathrm{~cm}$. The operation started in the septic chamber where the primary treatment was performed with biochemical processes, after entering the ditches, the water passed through a secondary treatment where they were filtered by the gravel and by the sand adhering to them the colloids, generating the decrease of the turbidity and therefore of the microorganisms. When having contact with the soil, the pathogens of the waste water died due to changes in temperature and humidity, also due to lack of nutrients, action of fungi and consumption by protozoa. The water treated in both trenches did not exceed the maximum permissible limits (LMP) according to the D.S. $\mathrm{N}^{\circ}$ 003-2010-MINAM and was within the environmental quality standards (ECA) of D.S. $\mathrm{N}^{\circ} 015-$ 2015-MINAM regarding the values of BOD, COD, conductivity, $\mathrm{pH}$, Dissolved Oxygen and Temperature. The total and thermotolerant coliforms were reduced by $90 \%$. The efficiency of the Niimi type trench was $70 \%$ and the normal trench was $61 \%$. The water treated by both systems complied with the LMPs and ECAs, however the Niimi type trench was more efficient.

Key word: waters, treatment, nutrients, quality, microorganisms. 
*Correspondencia de autor: km. 19 Carretera Central, Ñaña, Lima. E-mail: jhoenmerttinoco@upeu.edu.pe INTRODUCCIÓN

Así mismo ahora también existe un gran problema en lo que respecta a los sistemas implementados para el tratamiento de aguas residuales, ya que la mayoría de las veces estos sistemas no se encuentran en condiciones óptimas de funcionamiento, debido principalmente a que el diseño y la operación no se realiza adecuadamente en la gran mayoría de los casos, lo que hace muy importante la evaluación constante de los sistemas de tratamiento de aguas residuales para garantizar el logro de los objetivos técnicos y económicos.

En términos generales, la escasez de agua en una región puede estar asociada con su limitada oferta natural, difícil acceso y contaminación. Cuando el consumo es muy grande y los impactos que se causan en el agua afectan su suministro o calidad, la demanda individual de los sectores, incluyendo la de los ecosistemas, no puede satisfacerse completamente (Oliveira, 2008).

La implementación de la ecoeficiencia se inicia con la mejora de los procesos energéticos. Esta eficiencia aplicada al tratamiento de aguas residuales no discrimina las tecnologías existentes o empleadas, sino propicia mejorar la articulación de los componentes para crear operaciones y procesos que sean más económicos y sean ambientalmente amigables al ser empleados en la depuración de aguas residuales (Burga, 2016).

Las aguas residuales son aquellas aguas cuyas características originales han sido modificadas por actividades humanas y que por su calidad requieren un tratamiento previo, antes de ser reusadas, vertidas a un cuerpo natural de agua o descargadas al sistema de alcantarillado (OEFA, 2018). El tratamiento de las aguas residuales son aquellos trabajos encaminados a conseguir la eliminación o reducción hasta límites tolerables de sustancias contaminantes presentes en el agua, posterior a un uso que se haya dado, ya sea doméstico, agrícola o industrial (Alianza por el Agua)

Los procesos actuales para el tratamiento de aguas residuales pueden dividirse en cuatro etapas principales. El primero proceso es el tratamiento preliminar que se emplea para la eliminación de los sólidos en suspensión y los materiales flotantes, impuesta por los limites, tanto de descarga al medio receptor como para poder llevar los efluentes a un tratamiento secundario, bien directamente $\mathrm{o}$ pasando por una neutralización $\mathrm{u}$ homogeneización (Beltrán, Lora y Ramalho, 2003). El segundo proceso es el tratamiento primario que consiste en la eliminación de la materia insoluble como arenas, grasas y espumas del agua, siendo su primer paso la tamización o cribado, a través cámaras de sedimentación, también pueden usarse diversos procesos auxiliares, tales como flotación, floculación y tamices de malla fina donde se eliminan los sólidos grandes que entran en el sistema de alcantarillado (Veza, 2007). El tercer proceso es el tratamiento secundario que consiste en la conversión biológica de los sólidos disueltos y los orgánicos coloidales en forma de biomasa que luego son removidos por sedimentación. La conversión biológica la realizan los microorganismos que degradan los sólidos disueltos o los orgánicos coloidales, mediante la biooxidación. Finalmente el tratamiento auxiliar que abarca un gran número de procesos unitarios básicamente físicos y químicos que pueden usarse antes o después del tratamiento biológico secundario para cumplir con los objetivos específicos del tratamiento. 
Según Pastrán y Millán (2010) es uno de los sistemas más comunes de disposición in situ de agua residuales las cuales son utilizadas para descargar el efluente proveniente del tanque séptico $\mathrm{u}$ otro sistema de tratamiento previo, donde se realiza el proceso de depuración biológico en medio granular fino, en general arena aportada. Se trata de un proceso aeróbico; el oxígeno es suministrado mediante el paso del agua por el sistema de los intercambios gaseosos con la atmosfera.

Existen varios mecanismos para el tratamiento de aguas residuales, siendo uno de ellos, el uso de zanjas de infiltración. El tratamiento en el medio poroso de las zanjas se produce por la combinación de una serie de mecanismos físicos, biológicos y químicos. Si la inundación es permanente, el medio poroso actúa como un filtro anaerobio sumergido, mientras que, si la aplicación es periódica, actúa como un filtro percolador aerobio.

El sistema de disposición final de las aguas residuales puede utilizarse para el riego del área donde se localizarán los tubos perforados del campo de absorción ya que el terreno absorbe y utiliza el agua que está desechando (Pastrán y Millán, 2010).

El suelo se utiliza como un filtro y como reactor biológico aeróbico; es un sistema poroso que actúa sobre la carga contaminante principalmente mediante dos mecanismos: filtración superficial y oxidación biológica. Los mecanismos implicados permiten lograr tres objetivos principales de depuración: eliminación casi total de los sólidos en suspensión y de la materia orgánica particulada, oxidación de materia disuelta y transformación del nitrógeno (nitrificación); y reducción importante del número de microrganismos patógenos.

En las zanjas de infiltración, las superficies de infiltración son las dos paredes laterales y el fondo de la zanja. Sin embargo, antes de que las paredes laterales sean efectivas, es necesario que el desarrollo de la biopelícula en la superficie de fondo sea suficiente como para provocar el encharcamiento de la zanja. La capa biológica, se forma mientras que el efluente fluye continuamente hacia el suelo y los microbios digieren los componentes de las aguas negras. Dicha capa suele ser muy efectiva en la eliminación de virus y patógenos. Desde el punto de vista funcional, la biopelícula funciona como un filtro biológico. El grosor de la película biológica crece a medida que los microorganismos metabolizan la materia orgánica presente en el efluente (Rojas, 2010). El de tipo pluvial tiene una estructura más simple, se utiliza para disponer aguas pluviales.

El objetivo de la investigación fue determinar la eficiencia de las zanjas de infiltración tipo Niimi y normal en el tratamiento de aguas residuales domésticas en Villa los SaucesLurigancho a través de la cuantificación de la remoción de nutrientes (nitrógeno total, fósforo total, sólidos disueltos y turbidez) y mejora de las características fisicoquímicos y biológicas (temperatura, oxígeno disuelto, $\mathrm{pH}$, coliformes totales y fecales) del agua.

\section{MATERIALES Y MÉTODOS}

\section{ÁREA DE ESTUDIO}

Ñaña se encuentra situado en el distrito de Lurigancho - Lima y corresponde a una zona con clima muy seco -templado según los métodos de Thornthwaite. La zona de estudio está localizada en la Universidad Peruana Unión a una latitud sur de $11^{\circ} 59^{\prime}$ longitud 7650', al este de la ciudad de Lima a una altura de $647 \mathrm{msnm}$, ubicado al margen derecho 
del km 19.5 de la carretera central, perteneciente a la jurisdicción del distrito de Lurigancho Chosica - departamento de Lima (Figura 1).

Los análisis de los parámetros (coliformes totales y fecales) fueron realizados por el laboratorio de ensayo acreditado de la Universidad Nacional Agraria la Molina. Los otros parámetros (turbidez, $\mathrm{pH}$, conductividad, temperatura, oxígeno disuelto, $\mathrm{DBO}_{5}, \mathrm{DQO}$, fosfatos y sólidos totales disueltos) fueron analizados en el laboratorio de Ingeniería Ambiental perteneciente a la facultad de Ingeniería y Arquitectura de la Universidad Peruana Unión.

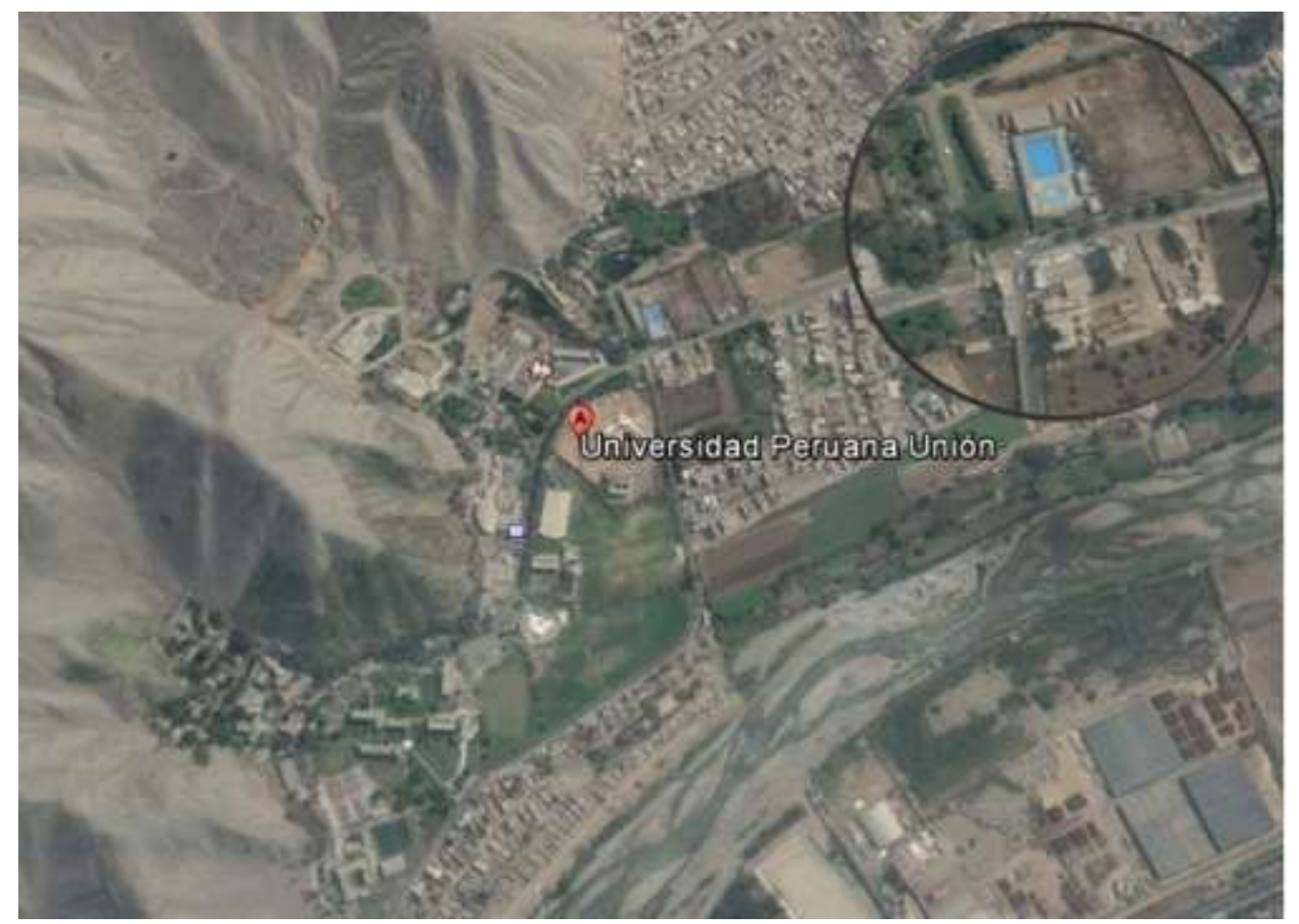

Figura 1. Mapa de ubicación de la zona de estudio

\section{PROCEDIMIENTOS}

\section{Descripción del canal abierto}

Se trata de un canal abierto, a la cual se vierten aguas residuales domésticas de las viviendas de las zonas aledañas al canal. El canal está situado a dos cuadras de la librería Betania, al frente de la Universidad Peruana Unión y al costado del terreno de cultivo de hortalizas.

\section{Muestreo y caracterización del agua de la vivienda}

Se ejecutó un muestreo simple, entre las 7:00 - 8:30 am del 15 de septiembre, siendo la hora puntal donde el caudal máximo horario tiene la mayor cobertura, continuidad y carga orgánica presente en dicha agua. 


\section{Características climatológicas de la zona de investigación}

La temperatura media anual de la zona es de $21^{\circ} \mathrm{C}$, el clima es húmedo-seco, la velocidad promedio del viento es de $6 \mathrm{~m} / \mathrm{s}$ principalmente en dirección a noreste (SENAMHI, 2018).

\section{Determinación del aforo del caudal}

Para el aforo del caudal en la acequia se utilizó para el aforo del caudal fue el método del flotador, debido a que es un canal abierto y que además de ello es un método de campo sencillo y rápido para estimar el caudal de agua que pasa en una sección transversal del rio; para la medición del caudal se realización los siguiente procedimientos: se determinó un punto A y un punto $\mathrm{B}$ y se midió la distancia entre ambos puntos, se medió la altura y ancho del canal, se liberó el flotador en el punto A y se cronometró el tiempo que demoró en desplazarse hacia el punto B y finalmente, se determinó el caudal aplicando la siguiente ecuación $\boldsymbol{Q}=\boldsymbol{V} * \boldsymbol{T}$. Dónde Q es el caudal $\left(\mathrm{m}^{3} / \mathrm{s}\right)$, V es el volumen (L) y T es el tiempo (min). En base a las mediciones realizadas se determinó una profundidad media de $24.1 \mathrm{~cm}$, un ancho de $130 \mathrm{~cm}$, largo de $1000 \mathrm{~cm}$ y el tiempo promedio de desplazamiento del flotador fue de 2.76 segundos. El área transversal fue de $2782 \mathrm{~cm}^{2}$.

\section{Aforo del caudal de entra a la zanja}

El caudal de entrada se calculó por el método volumétrico, para ello se empleó un balde de 20 litros de volumen ese determinó un tiempo de llenado de 30 segundo y un caudal de $0.66 \mathrm{~L} / \mathrm{s}$.

\section{Muestreo y análisis de la calidad del agua}

Para la determinación de la demanda bioquímica de oxígeno $\left(\mathrm{DBO}_{5}\right)$, fosfatos, conductividad, oxígeno disuelto, temperatura, turbidez, $\mathrm{pH}$ y solidos totales disueltos se utilizó tomaron muestras en frascos de plástico de $500 \mathrm{~mL}$, y para los parámetros microbiológicos (coliformes fecales y totales) la toma de muestras de agua fue tomada en frasco de vidrio de $250 \mathrm{~mL}$.

Los equipos empleados para el determinación parámetros fisicoquímicos fueron: multiparámetro Hanna para el $\mathrm{pH}$, conductividad eléctrica, solidos suspendidos totales y temperatura, oxímetro Hanna para la determinación de la concentración de oxígeno y turbidímetro Hanna para la determinación de la turbidez.

\section{Diseño de las zanjas de infiltración tipo Niimi}

Para el diseño y construcción de la zanja de infiltración tipo Niimi a nivel piloto, se tomaron en cuenta referencias bibliográficas.

a. Excavación de la zanja

Las zanjas de infiltración fueron determinadas de acuerdo con la estructura estándar. El ancho estándar fue de 30 a $50 \mathrm{~cm}$ y la altura de 50 a $70 \mathrm{~cm}$. La excavación de las zanjas de infiltración se hizo a mano, la que nos permitió observar las propiedades del suelo y el nivel freático. Finalmente obtuvimos una zanja sin asperezas e imperfecciones, con un 
ángulo recto en lo largo y en profundidad.

b. Relleno de la zanja

En la base se colocó plástico de $30 \mathrm{~cm}$ de ancho y largo de $250 \mathrm{~cm}$, sobre ella un tubo de $3 / 4$ " (Figura 23) con agujeros de $0.5 \mathrm{~cm}$ de diámetro cada uno a $4 \mathrm{~cm}$ de largo, las cuales fueron cubiertos con una malla de $30 \mathrm{~cm}$ de ancho y $250 \mathrm{~cm}$ de largo.

Después de colocar el plástico se rellenó hasta $10 \mathrm{c} 10 \mathrm{~cm}$ con arena de rio de diámetro de 0.1 a $1 \mathrm{~mm}$, la cual fue lavada y libre de material extraño e impurezas como basura o tierra. La grava capilar de 20 a $40 \mathrm{~mm}$ de diámetro, fue colocada encima de la arena, a una altura de 5 a $10 \mathrm{~cm}$, la colocación fue hecha a mano debido a que encima de este instalamos la tubería de alimentación y distribución de agua residual, la cual fue cubierta por una malla de $30 \mathrm{~cm}$ de ancho y $250 \mathrm{~cm}$ de largo. Finalmente fue cubierta con tierra arcillosa para realizar la siembra plantas de tallo bajo.

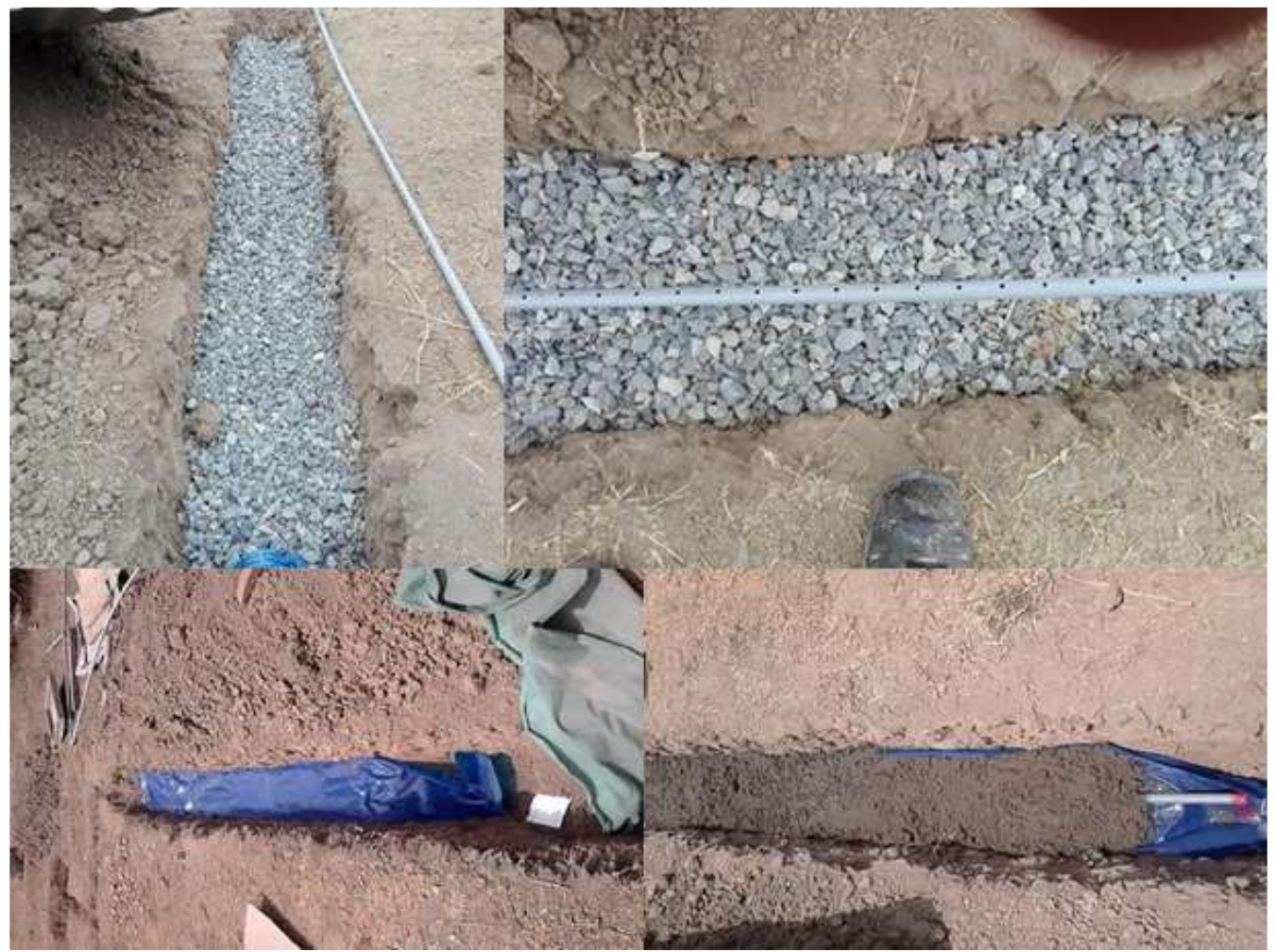

Figura 2. Rellano de la zanja tipo Niimi con arena fija y grava

\section{Diseño de las zanjas de infiltración Normal}

\section{a. Excavación de la zanja}

El ancho de la zanja fue de $30 \mathrm{~cm}$ y la altura de $50 \mathrm{~cm}$. La excavación de las zanjas de infiltración normal se hizo a mano, siendo que esta nos permitió observar las propiedades del suelo y el nivel freático. Finalmente obtuvimos una zanja sin asperezas e imperfecciones, con un ángulo recto en lo largo y en profundidad tal como lo muestra la Figura 3. 


\section{b. Relleno de la zanja}

En el relleno de la zanja de infiltración tipo normal, se colocó plástico de $30 \mathrm{~cm}$ de ancho y largo de $250 \mathrm{~cm}$, sobre ella un tubo de 3/4" (Figura 3) con agujeros de $0.5 \mathrm{~cm}$ de diámetro cada uno a $4 \mathrm{~cm}$ de largo, las cuales fueron cubiertos con una malla de $30 \mathrm{~cm}$ de ancho y $250 \mathrm{~cm}$ de largo.

Después de colocar el plástico se rellenó hasta los $10 \mathrm{~cm}$ con grava capilar de 20 a 40 $\mathrm{mm}$ de diámetro y encima de ella arena de río de diámetro de 0.1 a $1 \mathrm{~mm}$, la cual fue lavada y libre de material extraño e impurezas como basura o tierra. Posteriormente instalamos la tubería PVC $3 / 4$ " con agujero de $0.5 \mathrm{~cm}$ y cada uno a una distancia de $4 \mathrm{~cm}$ la cual sirve de alimentación y distribución de agua residual, la cual fue cubierta por una malla de $30 \mathrm{~cm}$ de ancho y $2.5 \mathrm{~m}$ de largo. Finalmente fue cubierta con tierra arcillosa para realizar la siembra plantas de tallo bajo (Figura 3).

\section{Diseño de tuberías y fuentes de alimentación}

Se construyó un andamio con una altura de $200 \mathrm{~cm}$ la cual sirvió para colocar el tanque de abastecimiento de agua residual, a este se le hizo la conexión de tubería de 1/2", instalando una llave de paso, a $60 \mathrm{~cm}$ de ella se colocó el tanque séptico, a la cual se le acopló una tubería PVC 3" de $250 \mathrm{~cm}$ de largo con reducción a $1 / 2$ ", a ella se le coloco un acoplas T, siendo a estas conectadas tuberías de $1 / 2$ " de $1 \mathrm{~m}$ de largo a, la cual tiene la función de distribuir agua residual a las dos zanjas de infiltración, a mitad de ella se colocó un llave de paso a cada una de los dos tubos, finalmente se hizo la unión de tuberías con codo de $1 / 2$ " las cuales se introducen hacia la zanja de infiltración tal como se muestra en la Figura 3.

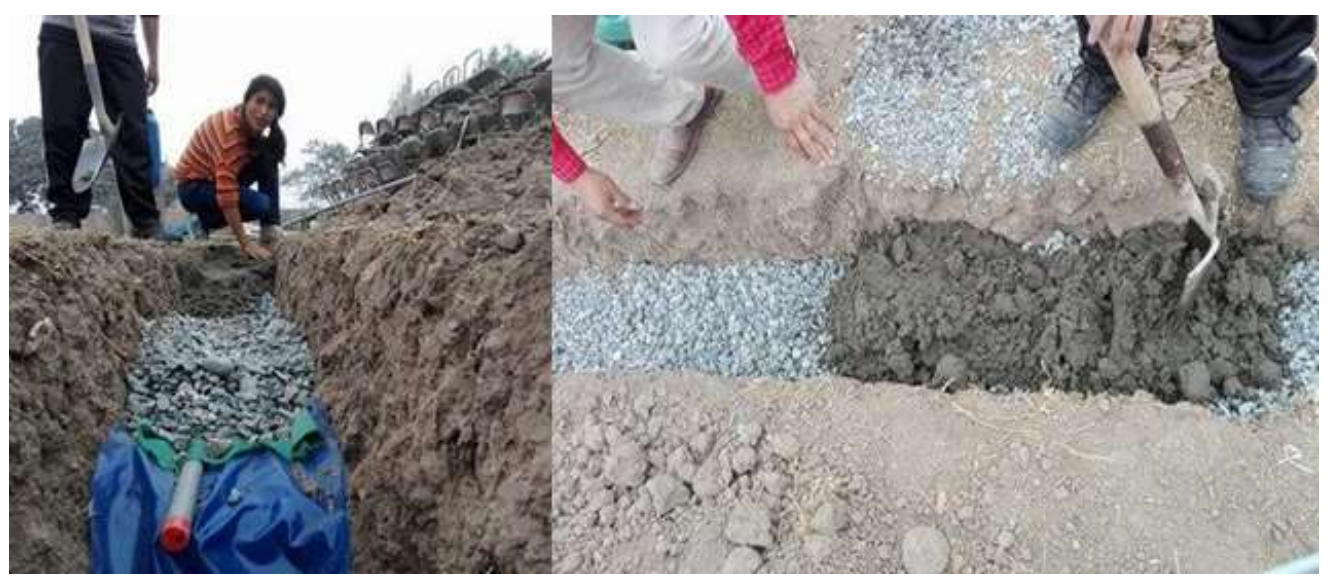

Figura 3. Relleno de la zanja de infiltración tipo Normal, primero grava, seguido de la arena fina.

\section{RESULTADOS Y DISCUSIÓN}

En los resultados se resume los datos recolectados y el análisis de los datos relevantes a fin de que pueda justificar las conclusiones. 
Los parámetros iniciales se realizaron en el Laboratorio de Ingeniería de la Universidad Peruana Unión y el laboratorio de Análisis de agua, suelo, Medio Ambiente y Fertirriego de la Universidad Nacional Agraria de la Molina.

Tabla 1. Resultados de los parámetros físicos, químicos y biológicos del agua de la zanja tipo Niimi

\begin{tabular}{|c|c|c|c|c|c|c|c|c|c|}
\hline \multirow{2}{*}{ Parámetros } & \multirow{2}{*}{$\begin{array}{c}\text { Pre- } \\
\text { análisis }\end{array}$} & \multicolumn{5}{|c|}{ Días de medición } & \multirow{2}{*}{$\begin{array}{l}\text { Mejor } \\
\text { resultado }\end{array}$} & \multirow{2}{*}{ LMP* } & \multirow{2}{*}{$\mathrm{ECA}^{* *}$} \\
\hline & & 1 & 2 & 3 & 4 & 5 & & & \\
\hline $\mathrm{T}^{\circ} \mathrm{C}$ & 21 & 20.1 & 25.3 & 24 & 20.8 & 23.4 & 23.4 & $<35$ & $\Delta 3$ \\
\hline UNT & 40 & 37.4 & 8.2 & 8 & 11 & 16.6 & 8 & - & - \\
\hline $\mathrm{pH}$ & 7 & 6.7 & 7.3 & 6.6 & 7.5 & 7.3 & 6.7 & $6.5-8.5$ & $6.5-8.6$ \\
\hline Oxígeno Disuelto (mg/L) & 0.7 & $\mathrm{~s} / \mathrm{d}$ & 8.75 & 9.03 & 9.75 & 10.09 & 10.09 & - & 4 \\
\hline Conductividad (uS/cm) & 500 & 470 & 610 & 640 & 540 & 520 & 470 & - & 2500 \\
\hline Solidos totals (mg/L) & 240 & 220 & 300 & 310 & 260 & 250 & 220 & - & - \\
\hline Fosfato $(\mathrm{mg} / \mathrm{L})$ & 2 & $\mathrm{~s} / \mathrm{d}$ & 2.75 & 2.75 & 2.31 & 0.49 & 0.49 & - & - \\
\hline $\mathrm{DBO}(\mathrm{mg} / \mathrm{L})$ & 30 & $\mathrm{~s} / \mathrm{d}$ & $\mathrm{s} / \mathrm{d}$ & 13.5 & $\mathrm{~s} / \mathrm{d}$ & $\mathrm{s} / \mathrm{d}$ & 13.5 & 100 & 15 \\
\hline DQO (mg/L) & 66 & $\mathrm{~s} / \mathrm{d}$ & $\mathrm{s} / \mathrm{d}$ & 30 & $\mathrm{~s} / \mathrm{d}$ & $\mathrm{s} / \mathrm{d}$ & 30 & 200 & 40 \\
\hline $\begin{array}{l}\text { Coliformes totales } \\
(\mathrm{NMP} / 100 \mathrm{~mL})\end{array}$ & 16000 & $\mathrm{~s} / \mathrm{d}$ & s/d & 1600 & $\mathrm{~s} / \mathrm{d}$ & $\mathrm{s} / \mathrm{d}$ & 1600 & - & 1000 \\
\hline $\begin{array}{l}\text { Coliformes termotolerantes } \\
\text { (NMP/100 mL) }\end{array}$ & 16000 & $\mathrm{~s} / \mathrm{d}$ & $\mathrm{s} / \mathrm{d}$ & 1600 & $\mathrm{~s} / \mathrm{d}$ & $\mathrm{s} / \mathrm{d}$ & 1600 & 10000 & 1000 \\
\hline
\end{tabular}

*Estándares de calidad Ambiental D.S. N 015- 2015-MINAM, **Límites Máximos Permisibles del D.S. N ${ }^{\circ}$ 003-2010-MINAM, s/d: sin dato

Tabla 2. Resultados de los parámetros físicos, químicos y biológicos del agua de la zanja tipo Normal

\begin{tabular}{|c|c|c|c|c|c|c|c|c|c|}
\hline \multirow{2}{*}{ Parámetros } & \multirow{2}{*}{$\begin{array}{l}\text { Pre- } \\
\text { análisis }\end{array}$} & \multicolumn{5}{|c|}{ Días de medición } & \multirow{2}{*}{$\begin{array}{l}\text { Mejor } \\
\text { resultado }\end{array}$} & \multirow{2}{*}{ LMP* } & \multirow{2}{*}{$\mathrm{EC} * *$} \\
\hline & & 1 & 2 & 3 & 4 & 5 & & & \\
\hline $\mathrm{T}^{\circ} \mathrm{C}$ & 21 & 20.1 & 24.1 & 20 & 20.1 & 23.7 & 23.7 & $<35$ & $\Delta 3$ \\
\hline UNT & 40 & 36.4 & 21 & 24 & 28.1 & 43.7 & 21 & - & - \\
\hline $\mathrm{pH}$ & 7 & 7.2 & 7.5 & 6.7 & 7.5 & 7.4 & 6.7 & $6.5-8.5$ & $6.5-8.6$ \\
\hline Oxígeno Disuelto (mg/L) & 0.7 & $\mathrm{~s} / \mathrm{d}$ & 9.5 & 2.97 & 8.77 & 9.47 & 9.5 & & \\
\hline Conductividad (us/cm) & 500 & 600 & 560 & 590 & 510 & 510 & 510 & & 2500 \\
\hline Solidos totals & 240 & 290 & 270 & 290 & 290 & 250 & 250 & & \\
\hline Fósforo & 0.652 & $\mathrm{~s} / \mathrm{d}$ & 0.9 & 0.9 & 0.37 & 0.4 & & & \\
\hline $\mathrm{P}_{2} \mathrm{O}_{5}$ & 2.99 & $\mathrm{~s} / \mathrm{d}$ & 4.11 & 4.11 & 1.67 & 1.82 & & & \\
\hline Fosfato & 2 & $\mathrm{~s} / \mathrm{d}$ & 2.75 & 2.75 & 1.12 & 1.22 & 1.12 & & \\
\hline DBO & 30 & $\mathrm{~s} / \mathrm{d}$ & $\mathrm{s} / \mathrm{d}$ & 13.5 & $\mathrm{~s} / \mathrm{d}$ & $\mathrm{s} / \mathrm{d}$ & 13.5 & & \\
\hline DQO & 66 & $\mathrm{~s} / \mathrm{d}$ & $\mathrm{s} / \mathrm{d}$ & 33 & $\mathrm{~s} / \mathrm{d}$ & $\mathrm{s} / \mathrm{d}$ & 33 & & \\
\hline Coliformes totals & 16000 & $\mathrm{~s} / \mathrm{d}$ & $\mathrm{s} / \mathrm{d}$ & 1600 & $\mathrm{~s} / \mathrm{d}$ & $\mathrm{s} / \mathrm{d}$ & 1600 & & \\
\hline Coliformes termotolerantes & 16000 & $\mathrm{~s} / \mathrm{d}$ & $\mathrm{s} / \mathrm{d}$ & 1600 & $\mathrm{~s} / \mathrm{d}$ & $\mathrm{s} / \mathrm{d}$ & 1600 & 10000 & 1000 \\
\hline
\end{tabular}

*Estándares de calidad Ambiental D.S. N $^{\circ}$ 015- 2015-MINAM, **Límites Máximos Permisibles del D.S. N $^{\circ}$ 003-2010-MINAM, s/d: sin dato

La turbidez es un parámetro que no figura en la categoría 3 de la normativa del D.S. $\mathrm{N}^{\circ}$ 015-2015-MINAM, ni en el D.S. N 003-2010-MINAM, según nuestros resultados (Tabla 1), el agua residual domestica ingresa con una turbiedad de 40 unidades nefelométricas de turbidez (UNT), al realizar el análisis pos-tratamiento en los 5 días, en la zanja de infiltración tipo Niimi se obtuvo un valor mínimo de 8 UNT en el día 3, demostrando una eficiencia de $80 \%$ y en la zanja de trinchera normal se registró una turbiedad de 21 UNT en el día 2 con una eficiencia de $47.5 \%$, esto se dio debido al materiales poroso tales como la arena y la grava que se pusieron en la zanja de infiltración. Yactayo (2004) menciona que cuando el agua turbia baja a velocidad a través de un manto poroso de arena, las impurezas entran en contacto con la superficie de las partículas del medio filtrante y son 
retenidas, desarrollándose adicionalmente procesos de degradación química y biológica que reducen la materia retenida a formas más simples, las cuales son llevadas en solución. La sedimentación permite que las partículas sean atraídas por la fuerza de gravedad hacia los granos de arena, lo que provoca colisión, este fenómeno se incrementa apreciablemente por la acción de fuerzas electrostáticas y de atracción de masas.

El pH inicial en la zanja tipo Niimi fue 7, y en los 5 días de muestreo de efluente tanto de la zanja de infiltración tipo Niimi y la normal varió 6.6 - 7.85, valores que están dentro de los aceptables según la normativa del D.S. N 015-2015 MINAM. Palomo (2015) menciona que la temperatura influye sobre el $\mathrm{pH}$ del agua, el agua pura tiene un $\mathrm{pH}$ de 7 solo exactamente a $25{ }^{\circ} \mathrm{C}$, a medida que la temperatura del agua sube, el $\mathrm{pH}$ disminuye; así mismo a mayor turbiedad se alcaliniza el agua. $\mathrm{El}$ pH incrementó en el cuarto día en ambos sistemas de tratamiento.

El agua residual ingresó con una conductividad eléctrica de $500 \mathrm{uS} / \mathrm{cm}$, después de pasar por la zanja de infiltración tipo Niimi en los 5 días de análisis de agua, se obtuvo un rango de $470-640 \mathrm{uS} / \mathrm{cm}$ donde el día 1 presentó una conductividad menor de $470 \mathrm{uS} / \mathrm{cm}$, con una eficiencia de remoción $6 \%$ y en la zanja de infiltración tipo Normal se adquirió resultados de rango $500-600 \mathrm{uS} / \mathrm{cm}$ siendo que los valores bajan con el tiempo, del cual en el día 5 se obtuvo un resultado mínimo de $500 \mathrm{uS} / \mathrm{cm}$ (Tablas 1 y 2). La conductividad del agua incrementa notablemente cuando se disuelven en ella sustancias ionizables, y los valores altos de conductividad corresponden normalmente a ecosistemas con elevada productividad biológica, además de ello a menor conductividad el agua es más pura (Morales, 2000).

La cantidad de sólidos disueltos totales en el agua, antes de ingresar al sistema de zanja de infiltración fue de $240 \mathrm{mg} / \mathrm{L}$, al realizar el análisis de agua de las dos zanjas en 5 días, el rango fue de $220-310 \mathrm{mg} / \mathrm{L}$ en la zanja Niimi, siendo en el día 1 el valor mínimo de 220 $\mathrm{mg} / \mathrm{L}$, con una eficiencia de remoción de $8.3 \%$, en la zanja Normal el rango fue de 250 $290 \mathrm{mg} / \mathrm{L}$, en el día 5 se determinó el valor mínimo de $250 \mathrm{mg} / \mathrm{L}$. La eficiencia es debido a la existencia de material tales como grava y arena las cuales cumplen de función de retener y además de ello se debe la degradación biológica de los microorganismos adheridos (Bernal, Sepulveda, Mathieu-Costello y Graham, 2003).

Según las tablas 1 y 2, la concentración de oxígeno disuelto en el agua de la zanja de infiltración fue de $0.7 \mathrm{mg} / \mathrm{L}$, al realizar análisis del efluente en 4 días en las dos zanjas de infiltración, en el zanja Niimi se obtuvo un rango de 8.75 - $10.09 \mathrm{mg} / \mathrm{L}$, donde en el día 5 se logró un resultado favorable de $10.9 \mathrm{mg} / \mathrm{L}$. En la zanja Normal se adquirió un rango de $2.97-9.47 \mathrm{mg} / \mathrm{L}$, siendo en el día 5 un máximo valor de $9.47 \mathrm{mg} / \mathrm{L}$. El oxígeno disuelto es considerado como un indicador de la calidad del agua, los valores normales varían entre los 7.0 y $8.0 \mathrm{mg} / \mathrm{L}$, además es un indicador de contaminación del agua y está relacionada con la capacidad del cuerpo de agua de ser soporte para la biota (Pérez, 2003). Generalmente, un alto nivel de oxígeno disuelto indica agua de mejor calidad, por otra parte, es esencial para indicar el estado de salubridad de los cuerpos de agua ya que da una idea de cuan contaminada está y cuanto puede ser soporte de vida vegetal y animal. La concentración del oxígeno disuelto en el cuerpo de agua está en relación a los cambios climáticos, turbulencia, temperatura y contenido de sales disueltas (Gómez, 2011).

La temperatura es el parámetro físico más importante del agua, porque afecta la velocidad de las reacciones químicas, la temperatura inicial en ambas zanjas fue $21^{\circ} \mathrm{C}$, después que 
el agua pasó por el sistema de tratamiento se realizó nuevamente análisis por un periodo de 5 días, donde en el sistema tipo Niimi la temperatura oscila de 20 a $23.3{ }^{\circ} \mathrm{C}$ y en el tipo Normal de 20 a $23.7^{\circ} \mathrm{C}$ (Tabla 1 y 2 ).

La temperatura de las aguas residuales es un parámetro importante, ya que afecta en las reacciones químicas y biológicas y la solubilidad de gases, como el oxígeno. En general las altas temperaturas elevan las tasas de reacción y solubilidad hasta el punto en que la temperatura se vuelve lo bastante alta como para inhabilitar la actividad de la mayoría de microorganismos (aprox. $35{ }^{\circ} \mathrm{C}$ ). (Diseño de plantas comunes de tratamiento de efluentes para aguas residuales industriales no peligrosas, 2002).

El análisis inicial del fosfato fue de $2 \mathrm{mg} / \mathrm{L}$, en los 4 días de análisis del agua residual de las dos zanjas, en la Niimi se obtuvo un rango de 0.49 a $2.75 \mathrm{mg} / \mathrm{L}$, siendo el día 5 con un valor mínimo de $0.49 \mathrm{mg} / \mathrm{l}$, con una eficiencia de $75.5 \%$ y en la Normal se obtuvo un rango de 1.12 a $2,75 \mathrm{mg} / \mathrm{L}$, en el cual en el día 4 se tuvo un valor más reducido de 1.12 $\mathrm{mg} / \mathrm{L}$ con una eficiencia de $44 \%$ (Tablas 1 y 2). Según Hernández (2011) los nitratos y los fosfatos provocan floraciones algales en las aguas superficiales, lo que hace que el nivel de oxígeno en el agua disminuya, provocando un estrés oxigénico debido a la toma de oxigeno por parte de los microorganismos descomponedores de algas.

El Fosforo inicial fue de $0.652 \mathrm{mg} / \mathrm{l}$, los resultados de análisis de agua de los dos tipos zanja de infiltración fueron las siguientes, en la zanja Niimi varió de 0.36512 a 0.8965 $\mathrm{mg} / \mathrm{L}$, siendo el día 5 la que presentó la reducción mínima de $0.0036512 \mathrm{mg} / \mathrm{L}$ y de la zanja Normal varió de 0.15974 a $0.8965 \mathrm{mg} / \mathrm{l}$, donde en el día 5 disminuye hasta los 0.15974 $\mathrm{mg} / \mathrm{L}$ (Tablas 1 y 2). El fosforo, uno de los nutrientes más comunes en el agua residual, se combinan con otros minerales presentes en el suelo obtiene una comunidad de bacterias, protozoos y hongos, que puede alimentarse de los nutrientes y de la materia orgánica del agua residual, cuando lo hacen desaparecen quedando está más limpia (Mariñelarena et al. 2006).

En una instalación bien diseñada, construida y mantenida, los nutrientes como el fósforo y el amonio serán absorbidos por las partículas del suelo mientras que los nitratos pueden migrar hasta los niveles freáticos.

El valor de DBO en el agua de las zanjas Niimi y Normal fue $30 \mathrm{mg} / \mathrm{l}$, y a la salida de la zanja de infiltración un valor de $13.5 \mathrm{mg} / \mathrm{L}$, se redujo notablemente (Tablas 1 y 2). Lo que indica un menor demanda en el día 3 dado a la menor carga microbiana.

El valor de DQO en el ingreso de los sistemas fue $66 \mathrm{mg} / \mathrm{L}$, y a la salida de la zanja Niimi se obtuvo un valor de $30 \mathrm{mg} / \mathrm{L}$ con una eficiencia de $54.5 \%$ y en la zanja Normal $33 \mathrm{mg} / \mathrm{L}$ con una eficiencia de remoción de 50\% (Tablas 1 y 2). Esta reducción se debe a la actividad biológica donde ocurren procesos químicos como la oxidación por microrganismos desnitrificadores en condiciones aerobias, los cuales descomponen todos los compuestos orgánicos reduciendo el oxígeno, convirtiendo en gas carbónico y agua donde una parte es absorbida por los microorganismos para la síntesis de nuevas células, la cual depende de la disponibilidad del oxígeno disuelto que existe dentro de la zanja (Burga, 2016).

Que además de ello se debe a los efectos combinados entre el tiempo de retención hidráulica, la acción filtrante del sustrato para retener solidos suspendidos, mediante procesos de la adsorción, a los materiales puestas tales como la grava y la arena fina las 
cuales sirven para la adhesión de bacterias degradadoras de materia orgánica y actúan como filtros de material particulado (Montoya, Ceballos, Casas y Morato, 2010).

La cantidad de Coliformes totales y termotolerantes el estudio fueron similares en ambos sistemas de tratamiento del agua, en el pre-tratamiento fue de $16 \times 10^{3} \mathrm{NMP} / 100 \mathrm{~mL}$, después de pasar por los sistemas de infiltración se obtuvo 16 x 10² NMP/100 mL (Tablas 1 y 2) donde la remoción de Coliformes termotolerantes fue de $90 \%$, el resultado no se encuentra dentro de los parámetros del D.S. N ${ }^{\circ}$ 015-2015-MINAM, la remoción se debe a que cuando el agua residual entra en contacto con el suelo estos patógenos mueren por los cambios de temperatura y de humedad, por falta de alimento adecuado, atacados por los antibióticos producidos por los hongos del suelo o comidos por protozoos. En una instalación bien diseñada, construida y mantenida, los patógenos serán destruidos y los sólidos filtrados (Mariñelarena et al., 2006). La variación se debe a la falta de condiciones nutricionales y ambientales que se desarrollan en el tanque séptico, ya que la mayor parte de la materia orgánica se degrada allí, y los patógenos que aun han pasado a la zanja de infiltración han sido retenidos mediante procesos físicos como la filtración y la adsorción las cuales son llevadas a cabo a través del medio de soporte (grava y arena fina), como anteriormente mencionado existen los patógenos ingresados suelo son depredados por los antibióticos producidos por los hongos del suelo o comidos por los protozoos (Burga, 2016).

Los resultados del análisis pos-tratamiento de las zanjas de infiltración Niimi y Normal, se encontraron dentro de los Límites Máximos Permisibles (LMP) de efluente para vertidos de cuerpos de aguas D.S. N$^{\circ}$ 003-2010-MINAM, estos resultados muestran una mejora en la calidad del agua con una eficiencia del $100 \%$, con ello se llega a una conclusión de que las zanjas de infiltración son confiables y económicas para el tratamiento de aguas residuales.

Teniendo los resultados post del análisis de los parámetros fisicoquímicos, del tratamiento con el sistema de las zanjas de infiltración, se hace una comparación con Estándares de Calidad Ambiental para el agua riego de vegetales (tallo alto y bajo) D.S. N $^{\circ}$ 015-2015MINAM - Categoría 3, los resultados analizados muestran que se encuentran dentro de los parámetros establecidos a excepción de los parámetros microbiológicos sobrepasan los valores dadas por la norma.

\section{RECOMENDACIONES}

El el diseño considerar una pendiente elevada, para que exista mayor presión y así pueda llegar toda el agua residual al último punto de la tubería; y que la tubería seguida del tanque séptico sea de 3" o mayor, para que exista un mayor caudal de descarga así al llegar al reductor de $3 / 4$ " la presión se incremente. La tubería para obtener el efluente sea enterrada $10 \mathrm{~cm}$ más profundo de lo excavado, debido que la mayor parte del tratamiento se realiza en contacto con el suelo; finalmente, la cámara séptica este enterrada, debido a que es un tratamiento anaerobio. Escoger una zona alta en que no se forme charcos o se inunde cuando llueve. Mantener la mayor distancia posible desde el sistema de tratamiento a cuerpos de agua superficiales (lagunas o arroyos), a perforación de extracción de agua, a los límites del terreno y a las edificaciones propias de los vecinos.

\section{Agradecimientos}

Al Laboratorio de Ciencias Biológicas de la Universidad Peruana Unión por otorgar facilidades logísticas para el desarrollo de esta investigación. 


\section{Referencias}

Alianza por el Agua (sa). Manual de depuración de aguas residuales urbanas. Monográficos Agua en Centroamérica. Recuperado el 15 en setiembre del 2018 de: http://alianzaporelagua.org/documentos/MONOGRAFICO3.pdf

Beltrán, D. J., Lora, F. y Ramalho, R. S. (2003). Tratamiento de aguas residuales . Madrid: Reverté, S.A.

Bernal, D., Sepulveda, C., Mathieu-Costello, O., \& Graham, J. B. (2003). Comparative studies of high performance swimming in sharks I. Red muscle mophometrics, valcularization and ultrastructure. Unites States: University of california San Diego.

Burga, R. M. (2016). Tratamiento de aguas residuales domesticas a nivel familiar, con Humedales Artificiales de flujo subsuperficial Horizontal, mediante la especie macrófita emergente Cyperus Papyrus (Papiro). Lima .

Gómez, I. C. (2003). Saneamiento Ambiental . Costa Rica : Universidad Estatal a Distancia

Hernandez, A. G. (2011). Tecnologias para el tratamiento de aguas servidas. Mexico : Universidad Veracruzana.

Mariñelarena, A., Guido, A., Alcantara, V. M., Cristina, I., Sobrero, C., Laborde, J. y Slomka, G. (2006). Manual de autoconstrucción de sistemas de tratamiento de aguas residuales domiciliarias . Buenos Aires : FREPLATA.

Morales, J. V. (2000). Cucunubá: Modelo para un desarrollo sostenible . Fundacion Universidad de Bogotá Jorge Tadeo Lozano: Bogotá.

OEFA (2018). Fiscalización ambiental en aguas residuales. Recuperado en 05 noviembre del 2017 en: https://www.oefa.gob.pe/?wpfb_dl=7827

Olivera, E. R. (2008). Alternativas de recolección y tratamiento de aguas residuales domésticas para pequeeñas comunidades en el departamento de Sucre. La Paz: Universidad de Sucre.

Palomo, J. N. (2015). Manipulación y ensamblaje de tuberías . Madrid: Paraninfo.

Pastrán, N. A. y Millán, R. L. (2010). Metodología para el dimensionamiento de zanjas de infiltracion para el tratamiento de aguas residuales domésticas. Barcelona: Universidad de Oriente.

Pérez, G. R. (2003). Bioindicación de la calidad de agua en Colombia . Antioquia: Universidad de Antioquia.

Rojas, J. A. (2010). Tratamiento de aguas residuales: Teoria principios de diseño . Colombia: Nuevas Ediciones S.A. Saade, V. S., Rivera, R. R., Guzmán, J. E., Sandoval, F. P., Castro, J. E., Guerrero, L. A., \& Romero, R. C. (2009). Guía tecnica sanitaria para la instalación y funcinamiento se sistema de tratamiento individuales de aguas negras y grises. El Salvador: Ministerio de Salud Pública y Asistencia Social.

Veza, J. M. (2007). Ingenieria Ambienta: Fundamentos, entornos, tecnologias y sistemas de gestion. Esoaña : McGraw-Hill/Interamericana de España S.A.

Yactayo, V. M. (2004). Tratamiento de agua para consumo humano. Lima: CEPIS/OPS 